\title{
The Agency for Healthcare Research and Quality (AHRQ) Practice-based Research Network (PBRN) Relationship: Delivering on an Opportunity, Challenges, and Future Directions
}

\author{
Wilson D. Pace, MD, FAAFP, L. J. Fagnan, MD, and David R. West, PhD
}

Practice-based research networks (PBRNs) often lack sufficient funding to develop the underlying infrastructure necessary to conduct high-quality, pragmatic, policy-relevant studies. One mechanism introduced by the Agency for Healthcare Research and Quality (AHRQ) that held the potential to address this issue was the PBRN Master Contract Program. The program allows the AHRQ to fund tightly focused "research activities" and to create a partnership through the PBRN contracts. Although PBRNs expected Master Contracts to strengthen them, several issues limit the utility of these contracts. The funding levels are lower than that provided from other sources for comparable work. Although some Task Order Officers are diligent, responsive, and supportive, too frequently their zeal for specific results and heavy handed approaches have led to significant "scope creep" and unrealistic expectations. Finally, a mechanism to allow PBRNs and network clinicians to influence the direction of the research questions has not been well developed. We see value in a new approach that supports the ability of the AHRQ to (1) garner support from other government agencies to engage PBRNs in studies relevant to policymakers and PBRNs; (2) capitalize on the collaborative nature of PBRNs by developing projects that support collaboration; (3) provide modest funding for infrastructure; (4) avoid the unnecessary and costly regulatory oversight from OMB; and (5) develop sustained "lines of research" on a scale, currently unavailable through the Master Contract, that can meaningfully contribute to the shaping of health policy. (J Am Board Fam Med 2011;24:489-492.)

The last decade has seen a remarkable growth in primary care practice-based research networks (PBRNs). In the late 1990s, when the Ambulatory Sentinel Practice Network (ASPN) ${ }^{1}$ attempted to form a PBRN collaborative, it could locate 12 po-

This article was externally peer reviewed.

Submitted 3 March 2011; revised 18 May 2011; accepted 20 May 2011.

From the American Academy of Family Physicians National Research Network, University of Colorado Denver Department of Family Medicine, Aurora, Colorado (WDP); Oregon Rural Practice-based Research Network (ORPRN), Department of Family Medicine, Oregon Health and Science University, Portland, Oregon (LJF); and Colorado Health Outcomes Program, Aurora, Colorado (DRW).

Funding: none.

Conflict of interest: The authors have each received contracts through the AHRQ PBRN Master Contract initiative and grants from AHRQ.

Corresponding author: Wilson D. Pace, MD, FAAFP, GreenEdelman Chair for Practice-based Research, Director, American Academy of Family Physicians National Research Network, University of Colorado, Denver Department of Family Medicine, 12631 East $17^{\text {th }}$ Avenue, MS F496, Aurora, CO 80045 (E-mail: Wilson.Pace@ucdenver.edu). tential member networks. By 2008, the Agency for Healthcare Research and Quality (AHRQ) PBRN registry included more than 100 such networks. ${ }^{2}$ Much of this growth can be attributed to support, both financial and intellectual, from the AHRQ. In 1999, the AHRQ began providing infrastructure support to help develop and expand PBRNs. Small infrastructure support grants led to more investigator-initiated research opportunities and the Primary Care-PBRN Master Contract. AHRQ has helped secure additional PBRN support from the National Institutes of Health (NIH), the Centers for Disease Control and Prevention (CDC), and from private foundations. Many of the PBRNs represented in this theme issue of the $7 A B F M$ have received AHRQ support and many of the manuscripts were supported through AHRQ funding.

With support from the AHRQ, PBRNs have matured as structures that are ideally suited for health care transformation, dissemination, and implementation research. ${ }^{3,4}$ PBRNs are well aligned to expand the knowl- 
edge base concerning the redesign of primary care systems to meet the needs of a reformed, high-quality, high-efficiency health care system. For PBRNs to fulfill their potential, the ongoing relationship between the AHRQ and PBRNs must prosper.

\section{The Current PBRN Research Business Model Is Not Sustainable}

We are concerned that the ability of PBRNs to fulfill a significant research role is being jeopardized by the precarious sustainability of the primary care PBRN enterprise. PBRNs typically have little access to infrastructure dollars. Key factors contributing to this include the fact that PBRNs are typically quite general in their focus (rather than disease-based) and typically exist in primary care departments or divisions where margins from patient care revenue are small. Although finding funding for research projects is possible, the opportunities to allocate research funding to the infrastructure of the PBRN are rare or nonexistent. Research organizations are not able to exist solely on the direct research funds they generate, and PBRNs are no different. PBRNs are relationship based, spending considerable time and resources to recruit practices and clinicians to join. ${ }^{3,4}$ Maintaining these relationships with practice visits, convocations, and network communications represents additional costs. Few PBRNs have developed business models that allow them to survive, let alone prosper, on research grants alone. Even when facilities and administration rates ( $\mathrm{F} \& \mathrm{~A})$ are high, such as with federal grants, academic centers rarely return significant funds to the grantee for infrastructure development and maintenance. For the few PBRNs that exist outside the academic world, F \& A rates are generally considerably lower, resulting in a similar limitation on the funds available for ongoing development. Even with the recent infusion of modest funding through institutionalspecific Clinical and Translational Sciences Award (CTSA) activities, nothing on the horizon suggests that the overall resource picture for PBRNs will improve. This lack of operating capital severely limits the ability of PBRNs to advance the discipline or to develop the underlying research and technical infrastructure necessary to address the demand for high quality, pragmatic, policy-relevant studies to guide transformation and health care reform.

\section{AHRQ's PBRN Master Contract}

One mechanism introduced by the AHRQ that held the potential to address these issues was the
PBRN Master Contract Program established in 2007. This mechanism allows the federal government to tightly focus applicants for "research activities" and created a partnership with the funding agency in conducting the work. The purpose of the PBRN Master Contract program is to provide rapid turnaround research leading to new knowledge and information to improve primary care. In 2007, the AHRQ awarded Master Contract status to 10 PBRNs. To date, 21 task orders have been assigned to these master contractors. The task orders have addressed a range of topics, including practice redesign, quality measurement, patient safety, primary care linkages with community resources, and clinical topics such as methicillin-resistant Staphylococcus aureus and sleep apnea. Wethe authors of this editorial- have each benefited from the AHRQ PBRN Master Contract initiative. We have also learned some important lessons.

\section{PBRN Expectations}

The expectation of the PBRN master contractors was that the contract mechanism would strengthen PBRNs and develop a significant research portfolio to demonstrate the worth of the model. Unfortunately, PBRNs participating in the current AHRQ Master Contract perceive the award funding to be less than funding levels from other sources for comparable work (the authors have been involved in applications and/or funded projects within five AHRQ Master Contracts as well as working with the Center for Medicare and Medicaid Services [CMS] and the CDC on a "contract" basis). Though clearly tied to available funding, there is a substantial disconnect between reasonable "costs" for high-quality research and the funding provided. Many Task Orders under the AHRQ Master Contract have required the PBRN to partially subsidize the effort required to complete the Scope of Work. Low funding levels have also effectively prohibited widespread collaboration between networks, a frequent occurrence outside of Master Contract activities. Thus, the contracts may have further eroded the "financial stability" of an already stressed system and do not promote or expand collaborative efforts that can be leveraged into future funding from other sources.

\section{AHRQ Expectations}

The PBRN Master Contractors understand the rapid turnaround time and defined deadlines when con- 
ducting contract research, but recent task orders have provided unreasonably short time frames for completing a proposal. PBRN research typically involves conversations with collaborators, network scientific review groups, and network practices. It takes time to find collaborators, recruit interested practices, and write a quality proposal. The rapid response times can undermine traditional PBRN project development processes, thus weakening the relationship between the network research staff and network members.

\section{Collaboration Between Researchers and Task Order Officers}

The Master Contract relationship between PBRNs and AHRQ, requires that the work proceed according to a carefully prescribed schedule of deliverables. This arrangement is conceptually sound, and we do not take issue with ensuring that the federal government receives valuable work from the contract process. The degree of collaboration, support, and understanding of the research process, and continuity between the Task Order Officers and the contractors is highly variable across the AHRQ Master Contracts as well as between federal agencies. Our perception is that roles for Task Order/Contract Officers may not be clearly defined, thus leading to variability in their relationships with PBRNs. Clearly, some Task Order Officers are diligent, responsive, and supportive. However, too frequently, their zeal for specific results and heavy-handed approach to prescribing research methodologies have led to significant "creep" in the scope of work and unrealistic expectations of our research teams. This is particularly evident with Task Order Officers who are not familiar with practice-based research methods. These disconnects further stress the financial and professional resources of the PBRN community, which are generally not deep and have little reserve to deal with these issues.

\section{Addressing the Priority Research Questions for Primary Care Clinicians and PBRNs}

A mechanism to allow PBRNs and network clinicians to influence the direction of the research questions has not been well developed. However, in January 2011, the AHRQ has reached out to the PBRN Master Contractors to seek input into future projects. We applaud this effort. Others have highlighted the importance of this process when engaging community clinicians. ${ }^{3,4}$ Developing an informed pipeline of priority areas of inquiry will enhance the relevance of the Master Contract process. It may take time to find funding streams for these "bottom-up" research questions, but it is impossible to find funds for undefined problems. PBRN Master Contract holders must be able to view themselves as true stakeholders in the conduct of ground-breaking AHRQ-sponsored research.

\section{Looking Abead}

The PBRN community stands ready to assist the AHRQ to improve the nature of these funding mechanisms. We wish to ensure that the vision that created AHRQ-sponsored leadership for PBRNs is not lost in the effort to fund the policy-relevant research that we so sorely need to improve health care in our country. The work conducted under the AHRQ PBRN Master Contract program has demonstrated the ability of PBRNs to conduct rapid turnaround studies. We are interested in a continuation and expansion of the underlying concepts of the program, with revisions. We suggest that the AHRQ consider the development of PBRN "Center Grants" as a mechanism for continuing and expanding the relationship with PBRNs, similar to NIH support for cancer, asthma, and dentistry. (AHRQ P01, RFA-HS-11-005 represents a move in this direction.) We see value in an approach that supports the ability of AHRQ to:

(1) garner support from the CMS, the NIH, and the CDC to engage PBRNs in studies relevant to policymakers and PBRNs,

(2) capitalize on the collaborative nature of PBRNs by developing projects that support collaboration or include incentives for Center Grant holders who actively include other PBRNs in their projects,

(3) provide modest funding for the infrastructure of the PBRN core,

(4) avoid the unnecessary and costly regulatory oversight from OMB that is associated with contracts rather than grants, and

(5) develop sustained "lines of research" on a scale currently unavailable through the Master Contract that can meaningfully contribute to the shaping of health policy.

We look forward to the future contributions of PBRNs and to the strong relationship that has been built with the AHRQ. We believe that the nation's primary care PBRNs are ready to respond with appropriate attention. We are sure 
research reported in this PBRN theme issue will add to the current body of evidence that supports the value of PBRNs and the points that we have discussed here.

\section{References}

1. Green LA, Wood M, Becker L, et al. The Ambulatory Sentinel Practice Network: purpose, methods, and policies. J Fam Pract 1984;18:275-80.
2. Agency for Healthcare Research and Quality. The AHRQ PBRN Resource Center (PBRN RC). 2010. Available from: http://pbrn.ahrq.gov/portal/server.pt?open= $512 \&$ objiD $=969 \&$ mode $=2$.

3. Ryan G, Berrebi C, Beckett M, et al. Reengineering the clinical research enterprise to involve more community clinicians. Implement Sci 2011;6:36.

4. Kahn K, Ryan G, Beckett M, et al. Bridging the gap between basic science and clinical practice: a role for community clinicians. Implement Sci 2011;6. 